Copyright-Ergänzung des Verlags transcript: This text is posted here by permission of transcript Verlag for personal use only, not for redistribution.

\title{
Das Bild als eigenständiges semiotisches System
}

\author{
Kunst- und kulturanthropologische Überlegungen
}

zu Mechanismen, Prozessen und Praktiken der

Bedeutungsgenerierung durch das bzw. im Medium Bild

\section{MARTINA SAUER}

\begin{abstract}
Kommunizieren wir über Bilder? Es ist das besondere Verhältnis von Bild und Text, das vor dem Hintergrund des Jugendstils in Illustrierten Zeitschriften um 1900 augenfällig wird, aber nicht nur in ihnen, das diese Frage zum Thema gerade der historischen Zeitschriftenforschung macht. Doch wie lassen sich Bilder in ihrer komplexen dynamischen Erscheinungsweise als Mittel der Kommunikation verstehen? Wie und was vermittelt sich uns über sie? Diesen Fragen aus bildwissenschaftlicher Perspektive nachzugehen, dient die nachfolgende Untersuchung. Einleitend gilt es dazu zunächst die These, dass wir tatsächlich über sie kommunizieren, am Beispiel eines Titelblatts aus der Jugend von 1896 aufzuzeigen. Mit der Auswertung der Forschungen zur Eigenlogik von Bildern, die bereits seit Mitte des 19. Jahrhunderts in der Kunstwissenschaft und nachfolgend in der Kulturanthropologie bewegt werden, gilt es die Annahme zu untermauern. Weiterführend sind es jedoch gerade deren Forschungsergebnisse zu den Grundlagen von Gestaltung und Wahrnehmung, die zur Erweiterung der These veranlassen, sich von der klassischen, erkenntnisorientierten, ästhetischen Theorie zu distanzieren und sie als kulturtheoretische, semiotische Theorie neu zu fassen. Die Neuausrichtung wird im Verständnis des Gehalts der Bilder im medialen Transfer greifbar: Statt in einer Ansicht von etwas (Erkenntniswert), so eröffnet sich, liegt er in einer Ansicht über etwas (Meinung/ Kulturwert).
\end{abstract}




\section{EinLEITUNG}

Vermag ein Bild neben der Veranschaulichung und der epistemischen Verbürgung des etwa in Zeitschriftenbeiträgen verhandelten Gegenstandes darüber hinaus als ein eigenständiges semiotisches System und Medium der Bedeutungskonstitution verstanden werden? Mit dieser Frage regten die Organisatorinnen des vorliegenden Bandes zu einem Nachdenken über Bilder an, sie in ihrer eigentümlichen komplexen, dynamischen Erscheinungsweise ernst zu nehmen. Trägt die lebendige Wirkung von Jugendstilbildern, deren freies Linienspiel uns spielerisch durch ihre zumeist harmlos erscheinenden Bildwelten geleiten, zur Generierung von Bedeutung bei? Bereits in der von mir nur in einem Satz beschriebenen Eigenart von Jugendstilbildern wird deutlich, wie schnell die Motivwelt und deren eigentümlicher Ausdruckswert, der auch die Illustrationen der Jugend, die seit 1896 in München erschien, ausmacht, als harmlos und gefällig abgetan werden kann. Dieser Auffassung entgegen, die von einer ästhetisch gestimmten Haltung geprägt ist, gilt es mit der nachfolgenden Untersuchung die wirkungsmächtige Eigenlogik der Bilder und deren Relevanz für die kulturelle Entwicklung herauszuarbeiten und Bilder entgegen der ästhetischen Theorie als eigenständiges semiotisches System vorzustellen.

Aus bildwissenschaftlicher Perspektive lassen sich konkret zwei Stränge aufzeigen, die dieser Annahme zuarbeiten. Der eine reicht bis zu den Anfängen der kunstwissenschaftlichen Forschungen zur formalen Ästhetik in die Mitte des 19. Jahrhunderts zurück. Neben Robert Zimmermann, Alois Riegl, Heinrich Wölfflin und Hans Sedlmayr sind es nach 1945 vor allem Max Imdahl, Gottfried Boehm und Lambert Wiesing, die diesen Ansatz aufgreifen. Der andere Strang geht auf die kulturanthropologische Forschungen Ernst Cassirers Anfang des 20. Jahrhunderts zur Frage der Generierung symbolischer Formen zurück, die - beeinflusst vom Pragmatismus - insbesondere von den amerikanischen Philosophen Susanne K. Langer und dem Mitbegründer von Bildakt 2008 an der Humboldt-Universität in Berlin John M. Krois weitergeführt wurden.

Beide Forschungsrichtungen, so lässt sich aufzeigen, heben darauf ab, dass die Eigentümlichkeit von Bildern weniger in deren potentiell abbildlich-wiedererkennbaren Qualitäten liegt, als in den letztlich abstrakten Formen. Demnach sind es die Farbverteilung und die Linienzüge, die etwa in den Illustrationen der Jugend sowohl die Rezeption als auch die Gestaltung ausmachen. Sie werden für die Generierung von Bedeutung als wesentlich angesehen. Möglich sei das, indem über die Variabilität der Formen und Farben unterschiedliche Ordnungen und damit Sinn gesetzt (formale Ästhetik) und darüber hinaus über deren angenommenes affektivvitalisierendes Potential der Betrachter erregt wird (kulturanthropologischer Ansatz). Im Ergebnis treffen sich beide Auslegungsrichtungen in der Annahme, dass 
mit der je individuellen formalen Struktur ein ästhetisches Erlebnis ausgelöst und die Bildaussage in charakteristischer Weise gesteigert wird.

Im Gegensatz zur klassischen ästhetischen Theorie, die in diesem Ansatz erkennbar wird, gilt es mit der nachfolgenden Untersuchung jedoch auf die darin liegenden kommunikativen Aspekte abzuheben und sie für das semiotische System Bild stark zu machen. Die mit der Ausarbeitung zu belegende These lautet entsprechend, dass über die Setzung abstrakt formaler und zugleich von uns affektiv aufgefasster Strukturen - über die beiden vorzustellenden Forschungsansätze hinaus Möglichkeiten der Wertsetzung und der Kommunikation von den jeweils mit den Bildern verhandelten Inhalten und Kontexten liegen. Über die dynamische Erscheinungsweise eines Bildes vermag derart ein Sinn kommuniziert werden, der weit über das harmlose, ästhetische Empfinden hinaus zu kulturell relevanten Entscheidungen und Handlungen veranlassen kann. Dem wie der Gehalt der Bilder im medialen Transfer übermittelt wird, gilt daher die nachfolgende Betrachtung. Sie zielt darauf, die Mechanismen, Prozesse und Praktiken der Bedeutungsgenerierung durch das bzw. im Medium Bild aufzuzeigen. Über deren Herleitung mittels einer konkreten bildlichen Analyse und der Auswertung der Forschung zum Thema, gilt es deutlich zu machen, dass über die abstrakten Strukturen und deren affektiv wirksames Potential nicht nur mehr oder weniger erkenntnistheoretisch relevante Inhalte vermittelt (ästhetische Theorie), sondern vielmehr die Auslegung eines Inhalts durch einen Produzenten kommuniziert wird (semiotische Theorie). Entsprechend wird mit der charakteristischen Verbindung von Form und Inhalt nicht nur das ästhetische Erleben angeregt und deren Zusammenspiel mit Lust oder Unlust aufgenommen, sondern, über das $>$ Was $<$ und $>$ Wie $<$ der Vermittlung vielmehr zu einer Stellungnahme aufgerufen, sei es zustimmend oder ablehnend. Über die funktionale statt traditionell ästhetische Auslegung von Bildern lassen sich, so die These, die handlungsrelevanten Potentiale der entweder nichtkritischen (affirmativen) oder kritischen (ablehnenden) Beurteilung dessen, was ein anderer geschaffenen hat, aufzeigen. Die Neuausrichtung ebnet den Weg, den erkenntnistheoretischen in einen kulturtheoretischen Ansatz zu überführen. Die bildwissenschaftliche Fundierung eines semiotischen Analysezugriffs auf Illustrierte Zeitschriften soll damit dem Status von Bildern als komplexe und dynamische Medien der Bedeutungskonstitution Rechnung tragen.

\section{EXEMPLARISCHE BILDANALYSE: ZUR JUGEND IN DER JUGEND (18. APRIL 1896)}

An die eingangs formulierte Annahme anschließend ist es nicht allein der verhandelte Inhalt, der für das Verständnis der Illustrationen der Jugend wesentlich wird. 
Im Gegenteil, dieser erscheint nach der ersten oberflächlichen Durchsicht der Illustrierten sogar wenig aussagekräftig, sondern vielmehr die Art und Weise, wie dieser umgesetzt wurde. Demnach ist es der Zusammenhang von Form (wie) und Inhalt (was), der, so die hier verfolgte These, grundlegend für die Konstitution von Bedeutung wird. Denn unabhängig von der Form, dem, wie das >Was< sich uns zeigt, ist diese Bedeutung nicht erschließbar. Die Form erst gibt dem Inhalt eine spezifische Ausrichtung bzw. einen Charakter. Daneben erscheinen uns die Illustrationen zudem gerade durch die charakteristische Linienführung lebendig bewegt, ein Phänomen, welches es mit der nachfolgenden Bildanalyse herauszuarbeiten gilt. Denn gerade diese Wirkungsweise, so gilt es weiterführend zu betonen, kann als ein Teil der Bedeutungsgenerierung angesehen werden. Auf diesem Doppelten und damit auf der Setzung abstrakt formaler und zugleich von uns affektiv aufgefasster Strukturen beruht das semiotische System Bild, so die Ausgangsthese. Übertragen auf den eher technischen Begriff, der es bewegenden Mechanismen, lässt sich präzisieren, dass dessen Antriebssystem nicht allein durch die Produzenten gesteuert, sondern nur von ihnen gesetzt wird und der Aktivierung durch den Rezipienten bedarf. In der Koppelung von Produzent und Rezipient mittels des Werks liegen damit die Möglichkeiten der Kommunikation nicht nur von Informationen, sondern - über deren affektiv-emotionale Bewertung durch uns - von Bedeutungen, die entsprechend angenommen oder verworfen werden können.

Mit der Beschreibung und Analyse eines Titelblattes der Jugend (Abbildung 1) soll dieser Zusammenhang anschaulich gemacht werden. Methodisch angelehnt an der klassischen kunsthistorischen Formanalyse gilt es deutlich zu machen, wie das >Was $<$ gestaltet wurde, indem konkret unterschieden wird zwischen dem, wie die Formen gegeben, die Farbe verteilt, die Körper hervorgehoben und der Raumeindruck erzeugt wurde. Aus ihrem Zusammenspiel lässt sich kunsthistorisch gesprochen auf die Komposition schließen. Doch entgegen der klassischen Beschreibungsmethode gilt es dabei zugleich sowohl auf der Was- als auch auf der WieEbene, deren jeweilige Erscheinungsweise hervorzuheben. Dabei handelt es sich um erste Interpretationen, über die deren zugleich kommunikativen Funktionen deutlich werden sollen (zur Methodik vgl. ergänzend Sauer 2000, 2012). So werden über die rein lokalen Beschreibungen der Motive hinaus, Rückschlüsse über deren mögliche Bewegungsabläufe, deren Situierung und deren Stellung zum Betrachter gezogen. Ergänzend gilt es bei der Beschreibung der jeweils gewählten bildnerischen Mittel, sie in Hinblick auf ihre Funktion auszuwerten. Wie werden über sie die Motive geformt bzw. festgelegt? Wie wird über sie der Raum definiert? Wobei zugleich deutlich werden soll, wie das Spiel zwischen den einzelnen Elementen ein Spannungsgefüge aufbaut, das wir jedoch weniger wahrnehmen als vielmehr nur empfinden können. Insofern soll das Augenmerk den »tensions and resolutions« gelten, wie es Susanne K. Langer formulierte (1967 [1953]: 206f.). Die Wirkungen des Zusammenspiels bestimmen schließlich, wie es mit der auf die kommunikativen 
Aspekte konzentrierten Beschreibung und Analyse herauszuarbeiten gilt, die Auslegung dessen, was mit ihnen vorgestellt wird, seien es die Motive oder auch nur die Bezüge im Raum bzw. zum Betrachter, der mit seiner Position vor dem Bild immer schon ein Teil dieses Beziehungsgefüges ist.

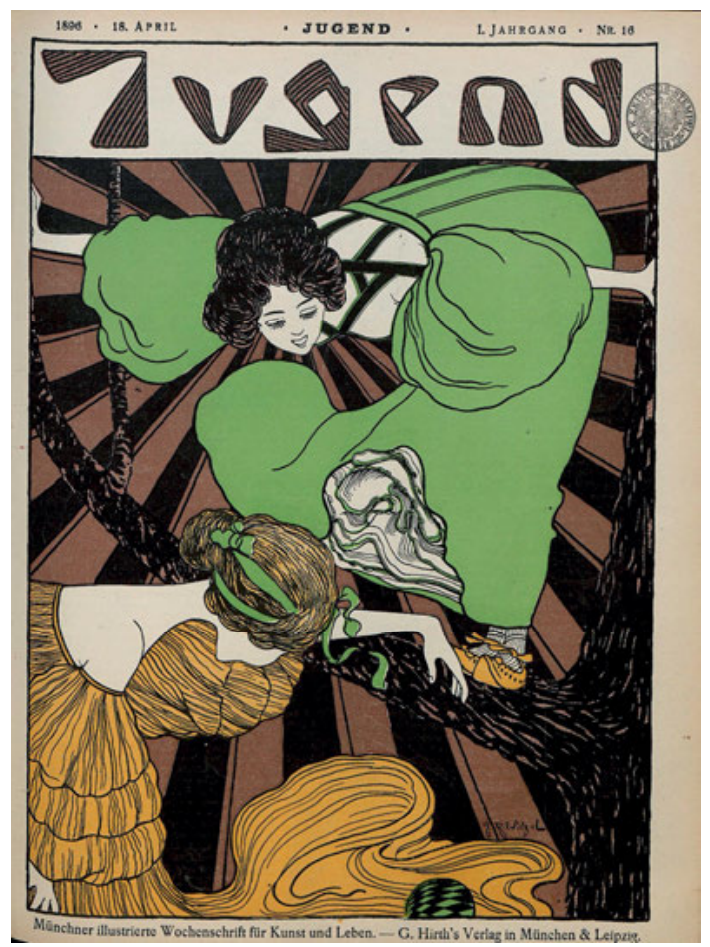

Abbildung 1: Illustration von J.R. Witzel. In: Jugend 1.16 (1896), Titelseite. Quelle: http://digi.ub.uni-heidelberg.de/diglit/jugend1896_1/0237 (letzter Zugriff: 29.04.2016); (C) Universitätsbibliothek Heidelberg.

Was wir auf diesem Titelblatt des ersten Jahrgangs der Jugend in der 16. Ausgabe vom 18. April 1896 sehen, ist eine Komposition mit zwei Figurinen: die schematische Darstellung einer von den Rändern beschnittenen Tänzerin unten links und einer zweiten, vollansichtigen, sich schwungvoll Vorbeugenden auf einem Ast darüber. Während erstere ins Bild tanzt, verneigt sich letztere vor dem Publikum und stützt sich dabei an den Rändern des Druckblatts ab. Durch die spezifischen angedeuteten Bewegungsabläufe und die Situierung der Tänzerinnen erweist sich das Publikum quasi als ein doppeltes: zum einen sind es die fiktiven Betrachter der Tänzerinnen, die durch das semantische >framing< aufgerufen werden; zum andern die $>$ realen $<$ Betrachter des Titelblatts der Jugend. 
Mit Blick darauf, wie diese dargestellt sind, handelt es sich um eine schematische Zeichnung, die die jeweilige Form der einzelnen Motive vornehmlich in scharf konturierten Umrissen und über die gleichmäßige, flächige Ausbreitung der Farben sowie wenige Binnenstrukturen festhält. Dieses kunsthistorisch als lineares System bezeichnete Verfahren der Formgestaltung (Wölfflin 1923 [1915]), betont entgegen einer tiefenräumlichen Entfaltung die Flächen- und damit die Nahwirkung. Es sind ergänzend streng geometrische Formelemente, wie die Rahmung des Motivs und ein als Fadenkreuz aufgespanntes Strahlensystem, die beide das flächige System stabilisieren. Diesen Tendenzen zur Verflachung und Verfestigung wirken die schwungvollen Gestaltungen des Schriftzugs Jugend und einzelner Binnenstrukturen sowie die ausschweifenden Gewänder entgegen. Das heißt, während die auf Geometrie und Fläche konzentrierten bildnerischen Mittel in ihrer Bewegungsfreiheit für uns stark eingeschränkt erscheinen, neigt das ausschweifende, kaum noch motivisch begründbare Linienspiel dazu, die strenge Bindung und verfestigende Wirkung auch für das eigene Empfinden aufzuheben. Denn letztlich sind es wir, die den Spannungen zwischen den einzelnen Elementen, der Flächenausbreitung und den Linienschwüngen mit unserem Blick nachfolgen und deren Zusammenspiel auch auf mögliche Bedeutungen hin mit unserem Blick realisieren.

Vergleichbare Beobachtungen lassen sich auch mit Bezug auf das gewählte Farbspektrum machen, das sehr reduziert ist. Es besteht aus einem hellen, matten Grünton und dessen Komplementär Rot sowie einem im Helligkeitswert und Intensitätsgrad abgestimmten Gelbton. Ergänzt wird die reduzierte Palette um die unbunten Farben bzw. Nichtfarben Schwarz und Weiß (bzw. als Papierfarbe eher Beige). Die jeweils monochrome und zumeist flächig ausgebreitete Verteilung der Farben betont ebenso wie die Formauffassung die Flächen- und damit Nahwirkung. Auch sie erscheinen derart in ihrer Bewegungsfreiheit stark eingeschränkt. Dem entgegen löst, wie zuvor das Linienspiel, nun die Wahl der Farben den reduzierten von der flächigen Ausdehnung und dem begrenzten Farbspektrum eingeengten Bewegungsspielraum auf. Denn Grün und Rot bilden einen natürlichen Komplementärkontrast, der bewirkt, wie in der Werbung erfolgreich praktiziert, dass die beiden Farben sich in ihrer Wirkung für uns gegenseitig steigern. Diese anfeuernde, die Aufmerksamkeit des Betrachters an sich ziehende Entscheidung wird durch die Verteilung der Farben unterstützt. Während das Grün und Gelb mit Weiß den Figuren vorbehalten ist, übernimmt das Rot mit Schwarz im Verbund mit den geometrischen Strukturen die Hintergrundgestaltung. Den einen mit dem anderen Bereich verbinden die schwungvoll gezogenen schwarzen Umrisslinien der Körper und Gewänder und die in Schwarz-Weiß changierenden, das Volumen und die Konturen der Frisuren und des Baumes nur andeutenden Binnenstrukturen. Dem flächigen und damit wenig Bewegungsspielraum gebenden Aufbau und der tendenziell frei lassenden, von der ornamentalen Wirkung der Einzelelemente getragenen Eindruck steht mit Blick auf die räumliche Ordnung das Fadenkreuz aus schwarzen und roten Strahlen entgegen. 
Diese laufen im Kopf der grünen Figurine zusammen und fokussieren damit den Blick im oberen Drittel und ziehen diesen zugleich unmittelbar in den Tiefenraum. Diesem Effekt steht jedoch die zuvor beschriebene ornamental organisierte Flächen- und damit Nahwirkung der Form- und Farbauffassung entgegen. Der tiefenräumlichen Ausdehnung arbeiten zudem auf motivischer Ebene die ausgestreckten Arme der grünen Figurine entgegen, mit denen sie sich rechts und links an den Rändern des Blattes festhält und sich damit mit tänzerischer Leichtigkeit dem Sog in die Tiefe entzieht. Dieses sowohl motivisch als auch formal wirksame Mittel löst sogar einen gegenteiligen Effekt aus: Mit der Verbindung zur Fläche, die diese harmlos ausgestreckten Arme der Figurine herstellt, vermag sich die Raumordnung in ihrer Wirkung plötzlich umzukehren. Vergleichbar der Optical Art, wird das Fadenkreuz plötzlich zu einem Pfeil, der nach vorne schießt.

Zusammenfassend gilt es vor dem Hintergrund der ausführlichen, auf formale Aspekte und deren Wirkungsspektrum konzentrierten Bildbeschreibung festzuhalten, dass mit Hilfe der eigentümlich eingesetzten bildnerischen Mittel (Linien, Flächen, Farben) eine außerordentliche Spannung aufgebaut wird. Diese entfaltet sich (1.) zwischen Fläche und Raum in der Umkehrung der Tiefwirkung, (2.) zwischen Senkrechten (Arme und Gewandbändern sowie den Blatträndern) und Diagonalen (Köpfe, Arme und Rücken/Baum, Gewand), (3.) zwischen einem gewundenen, ornamentalen Linien- und Farbenspiel (Gewand, Haare, Baum und aufbauschender Rock) und einem geometrischen Netz (Fadenkreuz und Träger des Gewands) und (4.) zwischen plastisch angedeuteten Elementen (Köpfen, Gewand der gelben Figurine, Baum) und flächigen (Gewand der grünen Figurine, sichtbare Körperteile, Fadennetz). Insofern ist es die Ordnung der abstrakten Elemente, der Linien, Flächen, Farben, die die Komposition ausmachen und denen zudem eine affektiv-vitalisierende Wirkung zugeschrieben werden kann, indem sie im Kontrast zu den je anderen eine lebendige, auf Spannungen beruhende Wirkung entfalten. Sie binden den Blick an die Fläche, gewähren ihm Spielraum mit den schwungvollen ornamentalen Elementen, fokussieren ihn, führen ihn schnell in die Tiefe und drängen ihn schließlich zurück nach vorne auf sich selbst zu. Von daher sind es die aufgebauten Spannungen, die das Hauptmotiv, die obere Figurine, in die Nähe des Schriftzugs $J u$ gend rückt und diese mit spielerischer Leichtigkeit trotz unsicherem Stand auf den Betrachter zuführen. Sie, die Jugend, symbolisch personifiziert durch zwei Tänzerinnen, steht im Fokus. Mit ihrer Kraft, mit ihren kreativen Ideen und ihrem von spielerischer, unbekümmerter Leichtigkeit geprägtem Tun verändert sie die Welt und damit uns, so lässt sich schließen.

Mit Bezug zur Ausgangsthese gilt es abschließend festzuhalten, dass das >Wie< entscheidend für das $>$ Was $<$ wird. Das semiotische System Bild beruht demnach, wie gezeigt werden sollte, auf abstrakt-formalen Bildelementen, aus deren Zusammenspiel wir auf Motive schließen und deren spannungsvolles expressives Miteinander von uns darüber hinaus als affektiv-vital wirksame Impulse erfasst werden. 
Damit gibt die Auswahl und Setzung der bildnerischen Mittel (Produktion) nicht nur vor, was wir sehen, sondern auch wie wir es verstehen sollen. In der Wahrnehmung (Rezeption) vermögen wir beide Aspekte einzulösen. Demnach sind es gerade die unspezifischen Aspekte der bildnerischen Strukturen, die nicht nur auf Wiedererkennbares hin ausgelegt, sondern deren auf Spannungen aufbauendes Wechselspiel von uns erfasst und affektiv-vital verarbeitet wird, die als Voraussetzung für die Generierung von Bedeutung im Bild verstanden werden müssen. Bedeutung meint hier einen Sinn, der über die inhaltliche Auslegung hinaus, deren Bewertung miteinbezieht. In diesem Doppelten, dem Deuten und Bewerten, wird die Funktionsweise der über die formal-abstrakte Struktur angelegten Bildlogik erkennbar, die dem Produzenten non-verbal die Kommunikation einer Botschaft ermöglicht. Die abstrakt-formale Bildanlage lässt sich insofern als eine Variable auffassen, über die die Bedeutung durch das Setzen inhaltlicher und durch das Anlegen affektivemotional bewertbarer Bezüge in je spezifischer Weise variiert bzw. ausgerichtet werden kann - eine Bedeutung, die entsprechend von dem Rezipienten verstanden und vor dem Hintergrund seiner soziokulturellen Prägung angenommen oder verworfen werden kann. Der in solcher Weise beobachtbare Prozess der non-verbalen Kommunikation des Produzenten über Bilder entpuppt sich unter Einbezug einer möglichen Antwort des Rezipienten als ein Dialog. Über die Analyse der Mechanismen (bildnerischen Mittel) und der Prozesse (der Wahrnehmung) wird schließlich eine Praxis erkennbar, nach der mittels der Reflexion des Verhältnisses der affektiven und wiedererkennbaren Bezüge der abstrakten Formen nicht nur eine Bedeutung erschlossen sowie ein Urteil, sei es affirmativ oder kritisch, gefällt werden kann, sondern über deren Konstruktcharakter auch die Medialität und damit die Aufgabe der Illustration, als ein Mitteilungsorgan zu fungieren, hervortritt. In Übereinstimmung mit literaturwissenschaftlichen Forschungen ließe sich auch in diesem Beispiel von einem Verfahren der Metaisierung sprechen:

Metaisierende Verfahren thematisieren oder potenzieren auf vielfältige Art und Weise den Fiktionscharakter von Literatur und anderen Medien oder weisen auf deren Künstlichkeit, Textualität und Medialität, d.h. deren Konstruktcharakter. Der gemeinsame Nenner der verschiedenen Spielarten der Metaisierung besteht darin, dass sie ihre jeweilige Form, Gattung oder das Medium zum Inhalt erheben. In funktionaler Hinsicht hingegen können sie sich erheblich unterscheiden und etwa über ein kritisches, spielerisches, parodistisches oder affirmatives Funktionspotential verfügten. (Hauthal et al. 2007: IX) 


\section{FORSCHUNGSSTAND: ZUR EIGENLOGIK DER BILDER IN KUNSTWISSENSCHAFT UND KULTURANTHROPOLOGIE}

\subsection{Formale Ästhetik}

Dass Form und Inhalt nicht voneinander getrennt gedacht werden können, ist eine Annahme, die bereits mit der Etablierung der Kunstgeschichte als Fach im 19. Jahrhundert von der formalen Ästhetik formuliert wurde. Der Kunsthistoriker Hermann Bauer hat diesen Zusammenhang in der Anthologie zu den Methoden des Fachs 1985 treffend beschrieben, indem er herausstellt, dass eine reine Analyse der Form ebenso unmöglich sei, wie die der Mitteilung, da beide nicht trennbar seien.

Sollte die Minimal-Definition von der Form als der Gestalt der Mitteilung richtig sein, ergibt sich, daß eine reine Formanalyse unmöglich ist, weil es unmöglich ist, die Mitteilung selbst nicht zu berühren. Nur von einer Mitteilung zu sprechen, geht ebensowenig an, denn die Mitteilung existiert nur in einer bestimmten Form. (Bauer 1988 [1985]: 158)

Der Philosoph Lambert Wiesing brachte dieses spezifische Verhältnis in seiner Untersuchung zur Geschichte der formalen Ästhetik, die in erster Auflage 1997 erschien, auf den Punkt, indem er im Anschluss an den Kunsthistoriker Heinrich Wölfflin herausarbeitet: Wahrnehmungsweisen und Gestaltungsweisen stehen in einer Analogie. Mit dieser Aussage wird auf etwas Grundlegendes hingewiesen, nämlich, dass beide, die Wahrnehmung und die Gestaltung auf ähnlichen Mechanismen aufbauen, sonst könnte das, was von den Produzenten als Mitteilung festgehalten wird und damit die Anschauungs- bzw. Wahrnehmungsweise von etwas, nicht in ein Bild umgesetzt und dann auch wieder verstanden werden. Formale Aspekte und solche der Anschauung bzw. Auffassung von etwas müssen demnach zusammenhängen. Abstrakte Elemente, wie sie die Form ausmachen, tragen insofern wesentlich zur Bedeutungsgenerierung einer Mitteilung bei. Über sie, so die Annahme, bekommt diese eine spezifische Gestalt und Ausrichtung.

Die Gesetzmäßigkeiten der immanenten Bildrelationen entsprechen den Anschauungsformen des Menschen. Die formale Ästhetik wird so in ihrem Selbstverständis durch Wölfflin grundlegend verändert. Denn dadurch, daß Wölfflin den Unterschied von Darstellungsformen und Anschauungsformen auflöst, unterwandert er die Opposition von Ästhetik als Theorie der Kunst und Aisthetik als Theorie der Sinne. Die formale Ästhetik tritt in den Dienst der Aisthetik, da Wölfflin das Bild verwendet, um die Wahrnehmung zu erforschen. Die Oberfläche des Bildes lässt Strukturzusammenhänge sichtbar werden, die auch als Prinzipien des Sehens aufgefasst werden können. (Wiesing 2008 [1997]: 18) 
Mit Blick auf das was sich zeigt, schließen die Forscher der formalen Ästhetik auf Präferenzen, die die Weisen, wie Welt gesehen wird, selbst betreffen. Diese liegen, wie es noch Robert Zimmermann (1854) in der Nachfolge von Johann Friedrich Herbart annimmt, in dem Ideal des Schönen. Sich von dieser Vorgabe lösend, unterstellt Alois Riegl (1901) ein Kunstwollen, das sich an haptischen oder optischen Aspekten orientiert, Wölfflin (1915) weist auf lineare oder malerische und Max Imdahl (1987) betont die Ausrichtung an gegenständlichen oder dynamischen Aspekten. Hier spannt sich eine Traditionslinie auf, die sich weg von der Beurteilung des ästhetischen bzw. harmonischen Spiels der Formen auf die Gestaltungsweisen selbst konzentriert, die entwicklungsgeschichtlich zwischen linearen und malerischen abzuwechseln scheinen (vgl. Wiesing 2008 [1997]: S. 25-205, sowie Prange 2004: 198-210). Zu ersteren zählt eindeutig das Titelblatt der Jugend, zu letzteren etwa die in Farbenwirbeln verschwimmenden Konturen der Segelboote William Turners (Abbildung 2).

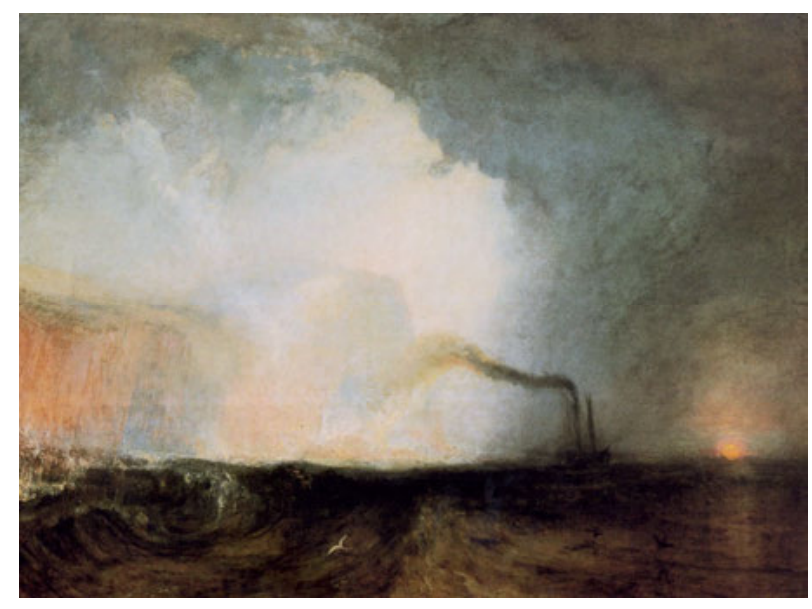

Abbildung 2: William Turner, Staffa, Fingal's Cave (1832). 91,5 x $122 \mathrm{~cm}$,

Öl auf Leinwand. Yale Center for British Art, Paul Mellon Collection. Quelle: http://www.zeno.org/nid/20004339800 (letzter Zugriff: 29.04.2016); gemeinfrei.

Innerhalb dieser Linie der formalen Ästhetik werden der Wahrnehmung selbst Präferenzen (im Sinn von Anschauungsweisen) unterstellt, insofern dass sie entweder eine lineare (haptische, gegenständlich orientierte) Gestaltung oder eine malerische (optische, dynamische Weltauffassung) bevorzuge. Sie spiegeln Interessen, die epochenübergreifend zu beobachten seien. Die wechselnden Stile können entsprechend als Ausdruck einer sich wandelnden Mentalitätsgeschichte bewertet werden.

Doch lässt sich über die historischen Präferenzen der unterschiedlichen Anschauungs- bzw. Gestaltungsweisen der Produzenten hinaus eine Aussage dazu ma- 
chen, was über die Werke jeweils über die Welt ausgesagt wird? In der Weiterführung der aufgezeigten Tradition ist es vor allem diese Frage, die die nachfolgende Forschung beschäftigt. Als Tenor kristallisiert sich dabei heraus, dass es die Wirklichkeit selbst sei, die über die formale Struktur in ihrer Wirkung gesteigert und damit etwas Wesentliches über sie ausgesagt werden könne. Das Werk erlaube damit über das ästhetische Erleben, die Welt je nach Präferenz der Produzenten (Stichwort Mentalitätsgeschichte) neu und anders wiederzugeben. Hierin liege dessen Vermögen. Bereits bei Konrad Fiedler lässt sich diese erkenntnisorientierte Tendenz herausstellen. Demnach sei es gerade dem Künstler möglich in einer ursprünglicheren Weise, in einer »Ausdrucksbeziehung zur Natur« (Fiedler 1887: 173), diese zu erfassen und in ein Werk umzusetzen. Methodisch findet dieser Ansatz in der Strukturanalyse, wie sie von der Sedlmayr-Schule Anfang des 20. Jahrhunderts begründet wurde, ihren Widerhall. In ihr steht, wie es Guido KaschnitzWeinberg in Abgrenzung zu Riegl formuliert, die »stete Verbundenheit mit dem zu erforschenden Objekt, das beständige Ausgehen vom gegebenen Kunstwerk selbst und nicht vom Subjekt oder von dessen Verhältnis zum Objekt wie bisher « (Kaschnitz-Weinberg 1963 [1929]: 34) und insofern der innere Wandel der Struktur von Körper- und Raumdarstellung im Hinblick auf das Wesen im Vordergrund. Erschüttert wird dieses neue Selbstverständnis durch die Entwicklungen der Moderne. Ausgelöst werden die Irritationen etwa durch Entwürfe für Gebäude in Kugelform wie etwa diejenigen von Etienne-Louis Boullée 1784 (Abbildung 3), in denen die Form nicht länger mit dem Inhalt bzw. der Wirklichkeitserfahrung übereinstimmt, die sich im Werk widerspiegeln soll.

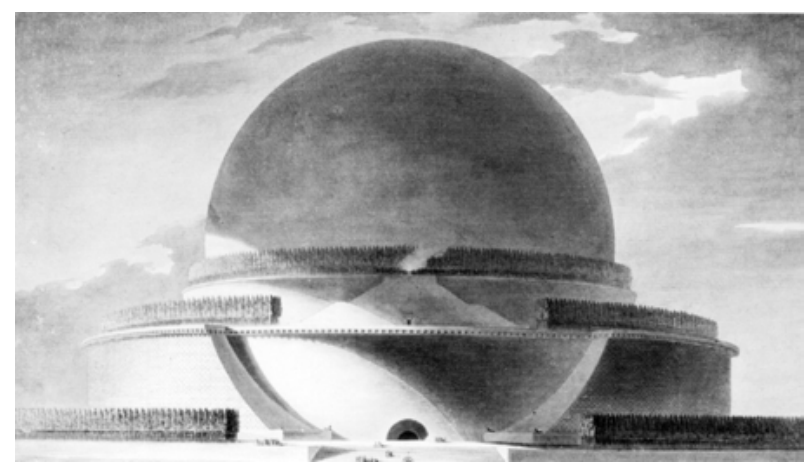

Abbildung 3: Étienne-Louis Boullée, Cénotaphe à Newton (1784). Entwurf für ein Isaac-Newton-Memorial. Quelle: http://commons.wikimedia.org/wiki/File:Newton_ memorial_boullee.jpg (letzter Zugriff: 29.04.2016); gemeinfrei.

Doch von was zeugen diese Entwürfe dann? Doch nur von einem Verfall, wie es der Kunsthistoriker Hans Seldmayr 1948 in seiner vielfach aufgelegten Schrift zum 
Verlust der Mitte behauptet. Damit weist er auf einen Verfallsprozess hin, der nicht nur in der Architektur, sondern in allen Gattungen der Kunst der Moderne zu beobachten sei (vgl. Sedlmayr 1985 [1948]: 10).

Eine Neuaufnahme und zugleich Neuausrichtung erfährt die Methode nach dem Krieg seit den 1970er Jahren. Internationale Bedeutung gewinnt sie im Anschluss an Imdahl durch den Kunsthistoriker und Philosophen Gottfried Boehm mit dem Ausruf des iconic turn in den 1990er Jahren und der Etablierung des Forschungszentrums Eikones in Basel seit 2006. Kritik an diesem Ansatz werden in jüngerer Zeit vor allem von den Visual Studies geübt, die sich an der nach wie vor tendenziell essentialistischen Auslegung entzündet (vgl. hierzu u.a. Schade/Wenk 2011: 35-53, Frank 2008: 477-487).

So bilden auch nach Boehm Form und Inhalt im Werk eine Einheit. Sie zeichne sich dadurch aus, wie er bereits 1978 wegweisend für seinen Ansatz herausarbeitet, dass Sein und Erscheinung zusammenfallen und sich entsprechend niemals vollständig sprachlich einlösen lassen. Dennoch werde gerade in diesem »abweisenden Schweigen des Bildes « sein tieferliegender Grund spürbar. Demnach weise dieses Schweigen auf einen »ikonischen Rest« eines anzunehmenden Ganzen hin. Das Besondere am Bild sei, dass dieser Grund »ganz Phänomen« wird. In seiner Phänomenalität sei er zugleich das »ikonisch Dichte«, weil in ihm alle Sinnrichtungen und Erscheinungsformen vereint sind (siehe Boehm 1985 [1978]: 454). Über das »ikonische Ordnungsmuster « der formal-abstrakt organisierten Struktur erschließe sich dieser Grund in Anlehnung an Imdahl in Simultaneität (dynamischen Prozessen) und Sukzession (gegenstandsorientierten Prozessen), wobei Boehm keine eindeutige Zuordnung der beschriebenen Prozesse zu linearen und malerischen Mitteln vornimmt (458, vgl. vertiefend Boehm 1980: 122-130): »Er [der Rezipient, M.S.] ist veranlasst, jenen Simultan- und Sukzessionsaustausch in Gang zu bringen. Jede einzelne Farbspur wird vor dem Hintergrund des Ganzen zur Auslegung einer Struktur, die sich im Prozeß der Zeitigung enthüllt wie verhüllt.«(Boehm 1987: 22) Entsprechend erlaube das, was sich mit ihnen zeige, »hindurchzublicken« auf eine Welt, in der wir uns schon immer bewegen und von der wir schon immer wissen im Sinn einer »primordialen Welthabe« (Boehm 2008: 21). Von der Fülle von Eindrücken, die uns der Erfahrungsgrund bietet, wählen wir, von der jeweiligen historischen Situation geprägt, sehend insofern immer nur bestimmte Aspekte aus. Der Wandel in den Darstellungsweisen lasse sich entsprechend als eine »Geschichte des Sehens« auslegen (vgl. hierzu Boehm 1985 [1978]: 466, Boehm 1992: 54f., 58-61). Entsprechend der Präferenzen des Sehens, die historisch bedingt sind, so wird auch in dem Ansatz Boehms deutlich, wird je neu und anders etwas von der Welt vorgestellt.

Hieran anschließend stellt sich grundlegend die Frage, hat das Bild einen Wahrheitsanspruch bzw. kann es als ein Mittel der Erkenntnis von Welt verstanden werden, wie es diese Tradition der formalen Ästhetik betont? Grundlegend wird dieser 
Anspruch von Lambert Wiesing abgelehnt, der dem entgegen zunächst die Produktion von »neuen Sichtbarkeiten« mit Bildern betont (vgl. Wiesing 2008 [1997]) bzw. vor dem Hintergrund semiotischer und phänomenologischer Forschungen von einem »Sehen lassen« der Produzenten durch Bilder ausgeht (vgl. Wiesing 2013). Meine eigenen Forschungen seit 2000, die unmittelbar auf der formalen Ästhetik aufbauen, lassen sich vor allem an diesen letzten Gedanken anschließen (vgl. Sauer 2000, 2012).

Mit Bezug zur Illustration der Jugend, wie sie eingangs vorgestellt und analysiert wurde, stellt sich die Frage, ob sich die hier aufgezeigten Forschungen zur formalen Ästhetik damit in Einklang bringen lassen. Spiegelt diese tatsächlich, wie es von Riegl bis Imdahl behauptet wird, eine lineare und damit am Haptischen und Gegenständlichen orientierte Anschauung von Welt wider? Und vermittelt sich über das Blatt etwas Wesentliches, das wir ohne es so nicht kennenlernen würden, wie es zuletzt Boehm annimmt? Auf der gleichen methodischen Basis aufbauend, legen die Ergebnisse der Bildanalyse, eine andere Auslegung nahe. Über sie werden Unterschiede zu dem erkennbar, welche Funktion der Produzent und Rezipient innerhalb des Theoriemodells übernimmt. So lässt sich mit Blick auf die Illustration zwar tatsächlich von einer Präferenz des Produzenten für eine lineare/haptische Darstellungsweise ausgehen, doch diese spricht eindeutig weniger von einem Bedürfnis, die reale Welt greifbar zu machen und sie zu veranschaulichen, sondern zeugt vielmehr durch die Reduzierung auf wenige Details wie den Haltungen, den Gewändern und Frisuren der Figurinen und der surrealen Situierung von einer überzogenen Stilisierung. Die These, dass diese lineare Darstellungsweise entsprechend weniger dynamische Aspekte betone, sondern statische, lässt sich so ebenfalls nicht halten. Im Gegenteil, das Liniengefüge gewinnt eine Eigendynamik, die sich für die Generierung von Bedeutung als wesentlich herausstellte. Mit Bezug zur inhaltlichen Aussage treffen sich die Beschreibungen in mancher Hinsicht mit der Boehms. Tatsächlich zeigt sich etwas von der Jugend, das so sicherlich nur diese Illustration zu zeigen vermag und sich nicht so leicht durch sprachliche Äußerungen ersetzen lässt. Doch auch wenn, mit Boehm, das »ikonisch Dichte«, das darin zum Ausdruck kommt, als ein Aspekt unseres gemeinsamen Erfahrungsgrundes ausgelegt werden kann, ist es tatsächlich das, was uns die Illustration vermitteln will? Erschöpft sich darin die Bedeutung?

Es sind die von dem Blatt selbst initiierten Reflexionsebenen, die, wie es an diesem Beispiel aufzuzeigen gilt, zu einer Ausweitung des von Boehm in der Tradition der ästhetischen Theorie vorgestellten, erkenntnistheoretisch orientierten Ansatzes hin zu einer semiotischen Theorie nahe legen. Statt in der Sphäre des ästhetischen Erlebens zu verbleiben und deren Beitrag für ein tieferes Verständnis von Welt fruchtbar zu machen, veranlassen diese den Blick zu weiten und den Einfluss des Bildes auf das Leben selbst, auf mögliche Entscheidungen und weiterführende Taten aufzuzeigen. Noch in Übereinstimmung mit der traditionellen ästhetischen The- 
orie ist es das Moment der Stilisierung und ästhetischen Steigerung bzw. die plötzliche Umkehrung des Raumerlebens und damit die Pointierung der Bildaussage, wie sie die Bildanalyse herausstellte, über die der Rezipient auf die Wirkungsweise des Mediums selbst aufmerksam wird und dessen Konstruktcharakter erkennen kann (metaisierendes Verfahren). Darüber hinaus werden jedoch zugleich die Möglichkeiten des gezielten Einsatzes der bildnerischen Mittel (Mechanismen), die Wahrnehmung zu steuern (Prozesse), offensichtlich. Gerade diese Möglichkeit verdeutlicht, dass sie auch dann bestehen muss, wenn sie nicht durch eine pointiert eingesetzte ästhetische Strategie aufgedeckt wird (vgl. hierzu ergänzend Sauer 2012). Insofern lassen sich mit Bildern Zwecke verfolgen, die nicht allein auf Werke der Propaganda und Werbung begrenzt sind, wie es die klassische ästhetische Theorie spätestens seit Kant annimmt, sondern auch solche der Kunst einbezieht (vgl. Kant 1991 [1790]: § 53). Deutlich wird das auch mit Blick auf die Bedeutung selbst, da dasjenige, was sich mit dem Werk zeigt, eher mit eigenen, den Produzenten zuschreibbaren Vorstellungen von Wirklichkeit, die nicht unbedingt allgemein gültig sind, übereinstimmt. Sie entsprechen, wie das Blatt der Jugend zeigt, einem bestimmten Kulturkreis, der nicht zwangsläufig mit anderen identisch sein muss. Über das Werk werden so gesehen von der jeweiligen Kultur geprägte, individuelle Bewertungen von etwas transportiert. Für die Bestimmung des Bildes als semiotisches System erweist sich diese Annahme als grundlegend, zeigt sich doch über sie, dass mit der Bildanlage nicht nur eine ästhetische Erfahrung ausgelöst, sondern vielmehr eine ästhetische Strategie verfolgt wird, in der es nicht allein um die gelungene Darstellung einer für alle gültigen, universellen Wirklichkeit geht, sondern um die Vermittlung von einer Ansicht (Meinung) über sie. Sie kann das Leben der Leserinnen und Leser bzw. des Publikums verändern (vgl. ergänzend Sauer 2015).

Von gänzlich anderen Voraussetzungen ausgehend, lässt sich die These, das Bild als semiotisches System aufzufassen, überraschenderweise noch aus einer zweiten Forschungsrichtung der Kunstwissenschaften, die sich zu Beginn des 20. Jahrhundert im Hamburger Umfeld um den Kunsthistoriker Erwin Panofsky etablierte, untermauern: von der Ikonologie. Sie baut auf kulturanthropologischen Überlegungen auf, die weniger von Panofsky selbst, sondern von dem Philosophen Ernst Cassirer entwickelt und nachfolgend von den beiden amerikanischen Philosophen Susanne K. Langer und John M. Krois vertieft wurden. Der von ihnen vertretene, auf kulturunabhängigen Voraussetzungen aufbauende Ansatz veranlasst sie, sich ergänzend auf Untersuchungsergebnisse aus den Lebenswissenschaften und solchen aus dem amerikanischen Pragmatismus zu stützen. Sie bilden die Basis für eine Verkörperungstheorie, mit der jedoch zugleich an der klassischen ästhetischen Theorie festgehalten wird. Dennoch erlaubt gerade deren Ansatz durch die konkreten Bezüge zur formalen Ästhetik, die darin erkennbar werden, das Verständnis vom Bild und dessen Funktion in unserer Lebenswelt zu präzisieren. 


\subsection{Kulturanthropologischer Ansatz}

Obwohl der Ikonologie vorgeworfen wird, sie vernachlässige mit der Erforschung des Inhalts und des Kontexts der Werke die formale Struktur der Bilder, geht auch sie davon aus, dass beide, Form und Inhalt, nicht zu trennen sind (vgl. Panofsky 1984 [1939]: 217). Wobei Panofsky dennoch ausdrücklich von einer formalen Betrachtungsweise im Sinn Wölfflins absieht, deren, wie er selbst sagt, »Analyse uns hier nicht weiter beschäftigen kann« und entsprechend nur zwischen einem »SachSinn « und damit Benennbarem und einem »Ausdrucks-Sinn « und damit Charakteristischem unterscheidet (vgl. Panofsky 1984 [1932]: 185-188, Panofsky 1984 [1939]: 210).

Vordergründig lässt sich hier tatsächlich zunächst kein Zusammenhang zur formalen Ästhetik herstellen. So werden die Grundlagen für den von Panofsky herausgestellten Ausdrucks-Sinn tatsächlich nicht, wie in der zu diesem Zeitpunkt das Fach dominierenden formalen Ästhetik in unterschiedlichen Anschauungsweisen von Welt gesehen, denen wechselnde Gestaltungsweisen entsprechen. Das heißt, statt davon auszugehen, dass es den Produzenten möglich ist, die Präferenzen, seien es nach Wölfflin malerische oder lineare mehr oder weniger frei zu wählen und zu variieren, nimmt die Ikonologie an, dass die Voraussetzungen des Menschen in charakteristischer Weise zu gestalten und den Ausdruckssinn von etwas zu erfassen, angeboren seien. Die Wahrnehmung selbst, so die Grundannahme, sei schon immer eine, die einen Ausdruck mitsieht. Entsprechend bezeichnet sie Ernst Cassirer als Ausdrucks-Wahrnehmung (Cassirer 1964 [1929]: 86). Entwickelt wird dieser Ansatz von Cassirer in den drei Bänden zur Philosophie der symbolischen Formen. Obwohl die Wahrnehmungstheorie darin eine zentrale Funktion einnimmt, verzichtet Cassirer jedoch darauf, sie explizit als Grundlage seiner Kulturtheorie vorzustellen. Stattdessen liegt der Schwerpunkt auf dem, was und wie der Mensch auf dieser Grundlage in Prozessen der Distanzierung mit Hilfe der Sprache (Bd. 1, 1924), dem mythischen Denken (Bd. 2, 1924/25) und seinen Erkenntniskräften (Bd. 3, 1929) eine Welt symbolischer Formen schafft. Auf dieser von Cassirer formulierten Kulturtheorie aufbauend gründet die Kunsttheorie der Ikonologie, deren selbst gewählter Schwerpunkt dann entsprechend auch auf der Analyse der je konkreten symbolischen Formen und damit dem historischen Kontext der Kunst liegt.

Da es jedoch gerade die hintergründige Wahrnehmungstheorie ist, die weiterführend auch von Langer und Krois aufgegriffen wird, die im Verbund mit dem Ansatz aus der formalen Ästhetik die Ausweitung der traditionellen ästhetischen Bildtheorie zu einer semiotischen Theorie erlaubt, gilt es sie im Folgenden näher vorzustellen.

In einer ersten Auseinandersetzung von mir zum Thema eröffnete sich bereits: Grundlegend für die Kulturphilosophie Cassirers wird die Annahme, dass wir alles, 
was wir wahrnehmen, schon immer dynamisch-bewegt erleben (vgl. Sauer 2009). Diese Weise Welt zu verstehen, mündet darin, dass sie für uns ausdruckshaft gesteigert erscheint. Diese ursprüngliche Wahrnehmungsweise bezeichnet Cassirer entsprechend als Ausdrucks-Wahrnehmung. Bestätigung fand Cassirer mit seiner kulturunabhängigen, anthropologisch argumentierenden Auffassung bei seinem Hamburger Kollegen dem Entwicklungspsychologen Heinz Werner, der in seinem lange Zeit als Standartwerk verhandelten Einführung in die Entwicklungspsychologie von 1926 festhält, dass der Mensch ursprünglich zur Welt kein distanziertes Verhältnis habe, sondern sie vielmehr als einen »vitalen Aktionszusammenhang « auffasse. Wobei die Wahrnehmung einer Gestalt über die Bewegungswahrnehmung aktiviert werde. Sie lasse sich bereits bei Tieren so beobachten (vgl. Werner 1959 [1926]: 38-44). Zusammenfassend schrieb Werner: »Diese physiognomische oder ausdrucksmäßige Betrachtung der Dinge ist bedingt durch die wesentliche Mitbeteiligung des affektiven dynamischen Gesamtverhaltens an der Gegenstandsgestaltung.« Die Welt werde daher weniger sachlich als ausdrucksmäßig, gesichthaft und lebendig erfasst. Das mache die ursprüngliche Schau von Welt aus, wie sie etwa auch Künstler haben, wobei Werner hier konkret den Maler Wassily Kandinsky anführt (vgl. 45-47, Zitat 46).

Daneben fand Cassirer jedoch auch in dem Hamburger Biologen Jakob von Uexküll einen Ansprechpartner, der ihm weiterführend eröffnete, dass die Wahrnehmung weniger visuell, sondern grundsätzlich senso-motorisch und damit körperlich erfolge (Propriozeption). Demnach wählt die Wahrnehmung nicht bewusst aus, was sie sehen möchte, sondern werde von der sie umgebenden Umwelt geprägt (vgl. Krois 2011 [2007]: 188-193). Daran anschließend sind es die Forschungen des mit ihm verwandten Neurologen Kurt Goldstein aus Frankfurt, die Cassirer veranlassten, schließlich zwischen einer unbewussten, natürlichen und einer bewussten, künstlichen Wahrnehmung zu unterscheiden. Der Umstand, dass die Fähigkeit zur Distanzierung bzw. bewussten Symbolisierung verloren gehen könne, während die natürliche, sinnliche Form der Symbolisierung, die sich im Tun äußere, noch erhalten bleibe, eröffnete ihm diesen Zusammenhang (vgl. Krois 2011 [1999]: 53). Cassirer übertrug diesen Vorgang in ein Modell von Leib und Seele, demzufolge von einer Transformation der natürlichen (sinnlichen) Zeichen in künstliche (kulturelle) ausgegangen werden könne (vgl. 43-62). Darüber hinaus stand Cassirer jedoch auch in einem unmittelbaren Austausch mit dem Kunst- und Kulturwissenschaftler Aby M. Warburg, dessen Bibliothek für ihn eine wichtige Quelle wurde. Von einem vergleichbaren, aber ins existentielle gesteigerten Gedanken geprägt, geht Warburg davon aus, dass wir der Welt nicht objektiv-neutral, sondern ihr schon immer mit Angst begegnen und sie mit Hilfe der Kunst bannen können, in dem Sinn von »Du lebst und tust mir nichts.« (Böhme 1997: 20, vgl. ferner Warburg 1992 [1923]) 
Einen konkreten Anknüpfungspunkt an die formale Ästhetik bieten die näheren Ausführungen Cassirers zu dem, was die Ausdrucks-Wahrnehmung ausmacht. Denn Cassirer verweist hier nicht auf wiedererkennbare Gegenstände, sondern auf abstrakte Bewegungsformen und Raumgestalten, die von uns schon immer affektivemotional ausgelegt werden. Cassirer hebt damit auf die richtungsgebende Qualität wahrgenommener abstrakter Elemente ab, deren je spezifische Erscheinungsweise als ein entsprechend dynamisches Geschehen ausgelegt werde:

>Wucht $<$, > Hast<, >Gehemmtheit< $<$ >Umständlichkeit<, >Übertriebenheit< sind ebenso sehr Namen für Lebenszustände wie für Bewegungsweisen und beschreiben in Wahrheit diese durch Angabe ihrer Charaktere. Wer Bewegungsgestalten und Raumformen kennzeichnen will, findet sich unversehens in eine Kennzeichnung von Seeleneigenschaften verstrickt, weil Formen und Bewegungen als Seelenerscheinungen erlebt worden sind, ehe sie aus dem Gesichtspunkt der Gegenständlichkeit vom Verstande beurteilt werden, und weil die sprachliche Verlautbarung der Sachbegriffe nur durch Vermittlung von Eindruckserlebnissen stattfindet. (Cassirer 1964 [1929]: 94)

Entsprechend charakterisiert Cassirer diese ursprüngliche Form des Wahrnehmens als eine, die durch ein Erleben und Erleiden gekennzeichnet sei. Dasjenige, was erfasst werde, erhält dadurch einen Ausdruck: »Ausdruck ist zunächst nichts anderes als ein E r l e i d e n ; ist weit mehr ein Ergriffenwerden als ein Ergreifen « (ebd.: 88). Dasjenige was über diesen Weg aufgegriffen wird, erhält derart eine Sinnrichtung.

Welche Bedeutung dieser Ansatz für die Wahrnehmung von Kunst hat, vermittelt bereits sein Austausch dazu mit Panofsky und Warburg. Jedoch erst in seiner Spätschrift An Essay on Man, die 1944 im amerikanischen Exil erschien, greift Cassirer das Thema selbst auf. Demnach werden gerade in der Begegnung mit Kunst, die ursprünglichen Ausdruckserlebnisse in reiner, potenzierter Form offenbar. Entsprechend der Natur ihres Wesens können die Bilder folglich zunächst weniger als Informationsträger, denn als Ausdrucksträger von spezifischen Erlebnissen verstanden werden. Cassirer interpretiert die Erfahrung mit den »lebendigen Formen« der Bilder dann jedoch nur - und hierin zeigt sich erneut die Nähe zur ästhetischen Theorie der formalen Ästhetik - als eine »Intensivierung der Wirklichkeit«. Exemplarisch verdeutlichte Cassirer diesen Zusammenhang, indem er die Erfahrungen mit einer natürlich schönen Landschaft »mit den Augen eines Künstlers« beschreibt:

[...] ich fange an ein Bild von ihr [der Landschaft, M.S.] zu formen. Damit habe ich ein neues Terrain betreten, das Feld nicht der lebendigen Dinge, sondern der »lebendigen Formen«. Nicht mehr in der unmittelbaren Wirklichkeit der Dinge stehend, bewege ich mich nun im Rhythmus der räumlichen Formen, in der Harmonie und im Kontrast der Farben, im Gleich- 
gewicht von Licht und Schatten. Der Eintritt in die Dynamik der Form begründet das ästhetische Erlebnis. (Cassirer 2007 [1944]: 233)

Über den Ansatz der formalen Ästhetik hinausgehend, verweist Cassirer damit auf ein Wahrnehmungsmodell, demzufolge nicht nur die Wahrnehmung von Kunst, sondern bereits die im Allgemeinen von abstrakten Strukturen bzw. deren als affektiv wirksam empfundenen Potentiale angeregt wird. Die Analogie, die aufgrund der abstrakten Strukturen zwischen beiden, den Wahrnehmungsweisen und Gestaltungsweisen gesehen wird, ist entsprechend bei Cassirer grundlegender als es die formale Ästhetik annimmt. Die abstrakten Strukturen werden nicht, je nach Präferenz in der Anschauungsweise von Welt sei es linear oder malerisch, mehr oder weniger bewusst gesetzt, sondern das Verhältnis kehrt sich um. Die abstrakten Strukturen bestimmen die Wahrnehmungs- und Gestaltungsweisen. So vermögen die abstrakten Strukturen über den Einsatz in der Bildproduktion, parallel zur ursprünglichen Wahrnehmung von Welt, sie erneut in gesteigerter Weise wiederzugeben. Das Tun des Produzenten und Rezipienten (das Gestalten mit und das Wahrnehmen von abstrakten, affektiv wirksamen Strukturen) entpuppt sich bei diesem Übertragungs- und dem dabei unterstellten Verstehensvorgang als wesentlich. Die Annahme, parallel zur formalen Ästhetik, die künstlerischen Praktiken vermitteln eine wahrheitsgetreue, im Ausdruck gesteigerte Wirklichkeitserfahrung, beruht darauf.

Im Anschluss an Cassirer ist es die amerikanische Philosophin Susanne K. Langer, die diesen Zusammenhang zum Thema ihrer Forschung macht. Noch bevor das Spätwerk Cassirers 1944 erschien, entwickelt sie einen eigenen kunsttheoretischen Ansatz, den sie 1942 vorstellt und in nachfolgenden Schriften systematisch ausbaut (vgl. hierzu Sauer 2014). Langers Aufmerksamkeit wendet sich entsprechend dem affektiv-emotional geprägten Erleben des Menschen zu, dass gleichermaßen bei der Betrachtung von Kunst aufgerufen werde. So sei der Gehalt der Kunst »das mit Worten nicht sagbare, und doch nicht unausdrückliche Prinzip der lebendigen Erfahrung, die innere Bewegungsform des empfindenden, seines Lebens bewussten Daseins.« (Langer 1965 [1942]: 252) Ihn zu verstehen, setze eine Vertrautheit mit der »impliziten « (und nicht diskursiven oder präsentativen) Bedeutung der Werke voraus, die eigener, »nichtdiskursiver« Formen des Begreifens bedarf (vgl. ebd.: 256-260). Die nicht-diskursiven Formen des Begreifens entsprechen, wie es bereits indirekt bei Cassirers erkennbar wird, formal-abstrakten Gestaltungsweisen. Letztere beruhen nach Langer in der Musik in der »tonalen dynamischen Form « und in der Malerei, Bildhauerei und Dichtung im »Spiel der Linien, Massen, Farben und Stoffe«. Wobei der Gehalt des künstlerischen Ausdrucks selbst, wie ihn Langer in dieser frühen Schrift noch als Vermutung nahe legt und in Feeling and Form 1953 zum Thema macht, in allen Künsten der Gleiche wie in der Musik sei (vgl. Langer 1967 [1953]: 103, 369 und 372). Entsprechend definiert Langer die Kunst als »the 
creation of forms symbolic of human feeling « (ebd.: 40). Die konkrete symbolbildende Kraft liege darin, dass über die Spannungen und Entspannungen der bildnerischen Mittel ein virtuelles Bild organischen Lebens entsteht (vgl. ebd.: 206-207, 47-59, 372). Sie beruhe auf der engen Beziehung zwischen organischen (leiblichen, M.S.) bzw. mentalen Prozessen (»vital forms«) und künstlerischen (»artistic forms «). Zwischen ihnen müsse, wie auch die formale Ästhetik ansetzt, eine Analogie bestehen. Darauf baut die (Bild-)Akt-Theorie Langers auf, wie sie sie in dem Doppelband Mind. A Philosophy of Human Feeling, dessen erster Band 1967 und der zweite 1972 erschien, herausarbeitet (vgl. Langer 1985 [1967]: 199-253). Erst im Zusammenspiel, so Langer, werden sie symbolisch (gefühlsmäßig) bedeutsam. Im logischen bzw. dialektischen Muster (»tension and resolution«) der möglichen Beziehungen (»potential acts«) forme sich der Sinn der Erscheinung als »lebendige Form« bzw. »illusion of bodily existence« (ebd.: 206).

Von Langer wird hier eine Erfahrung beschrieben, die so unmittelbar auch die Analyse des Titelblatts der Jugend aufdeckte. Doch in Erweiterung des von Langer vorgestellten Ansatzes gilt es nicht nur von einem »presentational symbolismus « $\mathrm{zu}$ sprechen, im Sinn der Präsentation von Gefühlen, die dem Rezipienten bewusst werden, sondern darüber hinaus von Bedeutungen bzw. Ansichten, die sich uns in Verbindung mit dem Motiv vermitteln. Diesen Zusammenhang hat Langer ebenfalls gesehen, ihn jedoch gerade für die Kunst ausgeschlossen. Die Kunst, so Langer, ermögliche eine Distanz, die eine auf Glauben-Machen und Delusion (Eins-Sein) ausgerichtete Gestaltung wie in der Unterhaltung und im Ritual nicht erlaube (vgl. ebd.: 319f.). So sei es gerade die Zweckorientierung der letzteren, die deutlich mache, dass sie nicht auf Gefühlen geprägten Erfahrungen mit der Welt anschließen (vgl. ebd.: 127f.). Doch genau besehen widerspricht diese Aussage ihrem eigenen Ansatz, wonach die Prozesse der Über- und Unterordnung von Akten (»tensions and resolutions «) nicht nur das Verhalten und damit die Entwicklung des Menschen ausmache, sondern alle Lebensbereiche betreffe. So seien sie bereits auf molekularer Ebene zu beobachten, die von physikalischen und chemischen Prozessen gesteuert werden. Eine These, mit der sie unmittelbar an ihrer Lehrer Whitehead anschließt (vgl. Whitehead 2000 [1927]: 122ff.). Mit der (Bild-)Akttheorie verweist Langer insofern auf einen universalen Mechanismus, der sich schließlich auch in organischen und psychischen und weiterführend künstlerischen Prozessen widerspiegle. Den Nachweis führt Langer über annähernd 900 Seiten, indem sie sich ergänzend wie zuvor Cassirer auf Forschungsergebnisse aus der Psychologie und Entwicklungspsychologie, Physiologie, Neurologie, Biologie und Zoologie stützt.

Funktional betrachtet, ermöglicht die Fundierung von Entwicklungsprozessen in dialektischen Akten demnach die Übertragung von handlungsrelevanten Informationen von einer auf die andere Ebene. Sie bewusst zu verstehen und entsprechend in Medien umzusetzen, kann demnach als die Basis für die Kommunikation und Weiterentwicklung des Menschen angesehen werden. Es sind inzwischen aktuelle ent- 
wicklungspsychologische und neurowissenschaftliche Studien, die diese Annahme stützen. Unter dem Stichwort Enaktivismus lassen sie sich greifen. Dazu zählen nicht nur die neurowissenschaftlichen Forschungen Vittorio Galleses, der 2007 gemeinsam mit David Freedberg bereits die Bezüge zur Kunst herstellte, sondern auch diejenigen des Entwicklungspsychologen Daniel N. Stern, der 1986 konkret sowohl an Langer als auch seinen Kollegen Werner anschließt (Stern 1992 [1986], Stern 2011 [2010]). Gerade dessen Ergebnisse wurden zuletzt in einem gemeinsamen Forschungsprojekt mit Giacomo Rizzolatti 2013 auch auf neurowissenschaftlicher Basis bestätigt (Guiseppe di Cesare et al. 2013).

Das (Bild-)Aktverständnis wie es Langer entwickelt, deckt sich in vielerlei Hinsicht mit dem des Cassirer-Experten John M. Krois (vgl. auch hierzu Sauer 2014). So ging auch er davon aus, dass es dem Menschen möglich ist, über die Künste an den Fluss des Lebens anzuschließen, der von Gefühlsbewegungen geprägt sei. Doch mit den Forschungen Langers hatte sich Krois nur am Rande beschäftigt (Krois 2011 [2010a]: 247f.), stattdessen schloss er mit seinen Überlegungen ebenfalls unmittelbar an Cassirer an. Bereits in der Schrift zu Cassirers Geschichtsauffassung 1987 und vertiefend in einer Reihe von Aufsätzen seit 1998, die 2011 von Horst Bredekamp und Marion Lauschke herausgegeben wurden, untersuchte Krois die Voraussetzungen für diese Annahme. Ebenso wie es bereits Langer betont, sei es die Kunst, die eine Objektivierung dieser Gefühlswelt erlaube: »In art, a medium permits giving expressive meaning an objective form. (Krois 1987: 132) Die expressive Bedeutung liege schon immer vor und sei grundsätzlich nicht-diskursiver Natur (vgl. ebd.: 59) Sie betreffe nach Krois die Wahrnehmung in ihrer ganzen körperlichen Gebundenheit: »Expressive meaning is not a product of culture; it characterizes the first stages of perception and bodily awareness [...] This is the prototyp of all symbolic relations. « (Ebd.: 57, vgl. ferner 85f.) Das ausdrucksmäßige Verstehen, wie es Cassirer einführte, kann daher, so lässt sich an die Ausarbeitung von Krois an früherer Stelle anschließen, als Funktion aller höheren symbolischen Formen (mit symbolischer Prägnanz) verstanden werden: Sie sei »the logical structure of experience« (ebd.: 47).

Doch erst spät, in seinem letzten Lebensjahr 2010, eröffnet sich dem Forscher konkret in Auseinandersetzung mit dem Enaktivismus (vgl. Krois 2011 [2010a]: 237f.), dass sich das Verstehen einer expressiven Bedeutung nicht nur mit der Willensbildung in Verbindung bringen lasse, und damit an die von ihr in einem Abwägungsprozess verfolgten Wünsche und Zwecke (»Evaluationsprozess«; vgl. Krois 1987: 155, 167, 102-105), sondern mit den Wahrnehmungsprozessen selbst zusammenhänge. So gewinnt die von Krois zuletzt in mehreren Aufsätzen angenäherte Verkörperungstheorie an Kontur. Sie gründet auf der Annahme, dass sowohl das Bild als auch der Körper (bzw. die Wahrnehmung des Menschen, aber auch von Tieren und Robotern) auf vergleichbaren Prinzipien bzw. Schemata aufbauen. Demnach besteht, so lässt sich zusammenfassen, zwischen den Bildschemata und 
Körperschemata eine Analogie: »Bei allen werden die Körperschemata aus den gleichen Bildschemata aufgebaut. Diese Bildschemata sind dynamische, nicht optische Formen [...].« Diese bewusst zu erleben und zu fühlen (als »Qualitäten«; Krois 2011 [2010a]: 231) über die »Ausdruckswahrnehmung (Krois 2011a: 270) zeichne den Menschen im Gegensatz zum Tier aus. Die Bezüge zu Cassirer, Langer aber auch Werner lassen sich hier deutlich erkennen. So sind es auch für Krois unbewusste, senso-motorische bzw. körperliche (Propriozeption) und damit nichtdiskursive Prozesse (des Körperschemas), die sowohl als Grundlage für eine bewusste, lebendige Selbsterfahrung (des Körperbildes) als auch für aktive Formbildungs- und Wahrnehmungsprozesse von Bildern und Zeichnungen (des Bildkörpers) angesehen werden können. (vgl. Krois: 2011 [2010a]: 231, Krois 2011a: 252271) Sie ermöglichen eine räumliche Orientierung und können daher als grundlegend für emergente und damit nicht auf Repräsentation beruhende, intelligente Handlungen angesehen werden. (vgl. Krois 2011 [2010a]: 231) Demnach sind es in Anlehnung an die Verkörperungstheorie von Charles S. Peirce (»philosophy of embodiment«; Krois 2011 [2009]: 198) die von ihm als »Qualitäten« (mit Peirce »icons «) bestimmten nicht-diskursiven, nicht optischen Formen, deren Veränderungen erfahren und ausgedeutet werden können. Doch Peirce sieht dieses Ausdeuten nur als einen Prozess des Denkens, eine letztlich unbewusste Verhaltensweise (Handlung) an, die die Verhaltensänderung erklärt (vgl. Peirce 1993 [1903]: 54-58, sowie 163-171), während Krois im Anschluss an Cassirer hierfür die AusdrucksWahrnehmung in Anschlag bringt, über die ein Ausdeuten der dynamisch, nichtoptischen Formen als gefühlte Qualitäten erfolgt (vgl. Krois 2011 [2009]: 204-209) und derart als unerlässlicher Anfang des Erkenntnisprozesses angesehen werden kann (vgl. Krois 2011c: 303f.).

Dennoch hielt Krois an verschiedenen Stellen fest - letztlich im Gegensatz zu seinen eigenen Aussagen -, dass auch wenn im Bild dynamische, affektiv wirksame Aspekte liegen, so seien diese unabhängig von den Intentionen der Produzenten und entsprechend auch der Interpretation der Rezipienten. Daher, so lässt sich mit Krois festhalten, ist das Bild keine Mitteilung und hat demzufolge auch keine Handlungsrelevanz: »The ursupatory character of pictorial objects - the fact that they possess affective meanings independently of the artists' intentions and the viewer's deliberate interpretations - results from the fact that like the viewer, they too embody dynamic affective image schemas.« (Krois 2011 [2010b]: 251, vgl. ferner Krois 2011a: 269, Krois 2011b: 278 und Krois 2011c: 306)

1 Wobei Krois hier keinen unmittelbaren Bezug zu den Bestimmungen dazu von Cassirer herstellt, die er jedoch an anderer Stelle vorgestellt hat (vgl. Krois 2011 [2006]: 142 und Krois 2011 [2007]: 162-174, hier 171). 
Grundsätzlich stellt sich auch vor dem Hintergrund der hier aufgezeigten kulturanthropologischen Forschungen die Frage, ob diese sich in Einklang bringen lassen mit dem, was eingangs am Beispiel einer Illustration aus der Jugend deutlich werden sollte. Tatsächlich, so zeigte sich bereits im Anschluss an die formale Ästhetik, wird mit der Bildanlage und deren Realisierung durch den Rezipienten, wie es Cassirer, Langer und Krois betonen, die Bildaussage, die Jugend zu symbolisieren, gesteigert. Doch lässt sich dieser Symbolisierungs-Prozess vom Produzenten abkoppeln, wie es zuletzt Krois und indirekt auch Langer und Cassirer behaupten? Wird die Sinnrichtung mit der Setzung durch den Produzenten nicht vorgegeben? Hier eröffnet sich ein Widerspruch in der Argumentation, den es näher zu betrachten gilt. Denn alle drei heben darauf ab, dass es die Erfahrungen des Produzenten mit der Umwelt sind, die für die Realisierung wesentlich werden. Demnach können abstrakt-formale und zugleich affektiv verarbeitete Aspekte der Umweltwahrnehmung - die »Bewegungs- und Raumformen« (Cassirer), die »tensions and resolutions « (Langer) und die »dynamischen, nicht-optischen Formen « (Krois) - analog in relational-logische und erneut affektiv wirksame Bildelemente umgesetzt werden.

Wahrnehmungs- und Gestaltungsweisen beruhen so gesehen auf denselben Prinzipien bzw. Mechanismen. Es sind daher keine Informationen, sondern immer schon bewertete Informationen, die vor dem Hintergrund des eigenen Kenntnisstandes und der kulturellen Bedingungen beurteilt werden. Darauf heben ausdrücklich bereits Cassirer und Langer ab. So habe gerade der Mensch im Gegensatz zum Tier die Möglichkeit zu »responses« statt»reactions«. Erst das mache ihn zu einem »animal symbolicum « (Cassirer 2007 [1944]: 49). Dem schließt sich Langer an, wenn sie betont, dass Tiere sich von den Situationen und Stimulationen der Umwelt nur anregen lassen (Langer 1972: 130f.), während es dem Menschen möglich sei, sie mit Vorstellungen und symbolischen Funktionen in Verbindung zu bringen (ebd.: 301-312). So sind es gerade sie, so gilt es weiterführend festzuhalten, die über die Bildanlage gesetzt und über die Wahrnehmung eines Anderen realisiert und vor dem Hintergrund der textlichen Bezüge sowie dessen Erfahrungen und dessen soziokultureller Situation beurteilt werden können. Dagegen mit Bezug auf Bilder bzw. Kunst davon auszugehen, der Produzent habe nicht die Möglichkeit die Bewertungen selbst vorzugeben und habe daher kein Ziel vor Augen, wenn er gestaltet, sondern vermittle selbstlos nur eine im Ausdruck gesteigerte Wirklichkeitserfahrung, so wie es auch die formale Ästhetik unterstellt, erscheint entweder zu instrumentalistisch oder zu idealistisch gedacht. 


\section{FAZIT UND AUSBLICK: DAS BILD ALS SEMIOTISCHES SYSTEM}

Lässt sich das Bild neben der Veranschaulichung und der epistemischen Verbürgung darüber hinaus als ein eigenständiges semiotisches System und Medium der Bedeutungskonstitution verstehen? Sowohl die Analyse eines Titelblattes der Illustrierten Zeitschrift Jugend vom 18. April 1896 als auch die Auswertung der Forschungen zur Eigenlogik der Bilder sollten das aufzeigen. Als Voraussetzung dafür kristallisierte sich heraus, dass der über die Eigenlogik kommunizierte Gehalt der Bilder nur über die Wahrnehmung des Rezipienten eingelöst werden kann. Er lässt sich nicht an den wiedererkennbaren Motiven ablesen. Voraussetzung dafür bildet die Annahme, dass die Wahrnehmung von Bildern nicht passiv, sondern durch ein Tun gekennzeichnet ist, über das die von den Produzenten gesetzte relationallogische Ordnung der abstrakten Strukturen als sinnvolle Informationseinheiten erfasst und zugleich über deren affektiv wirksames Potential als lebendiger charakteristischer Zusammenhang aufgenommen werden kann. Traditionell wird mit dieser Erfahrung des Rezipienten, das ästhetische Erleben verbunden, wie es auch von der formalen Ästhetik und den kulturanthropologischen Ansätzen vorgestellt wird. Eine entscheidende Wendung erfährt diese Auffassung, wenn davon ausgegangen wird, dass mit dem ästhetischen Erleben nicht nur eine Erfahrung der Wirklichkeit in charakteristischer Weise gesteigert, sondern vielmehr die jeweilige Auffassung davon kommuniziert wird, zu der sich der Rezipient stellen kann. In der Wendung von der Ansicht von etwas in eine Ansicht über etwas liegen die Nuancen, in denen der Wandel von der ästhetischen Theorie (Erkenntnistheorie) hin zum Bild als semiotisches System (Kommunikations- bzw. Kulturtheorie) ausgemacht werden kann.

Vorstellbar wird ein solches Modell letztlich nur, indem - wie von der Forschung indirekt vorgeschlagen - von universellen Wahrnehmungsprozessen und Gestaltungsmechanismen ausgegangen wird, die als Grundlage einer allgemeinen Kommunikations- und darauf aufbauend Kulturtheorie angesehen werden können. Diese besagt, dass das, was wir wahrnehmen abstrakt-formal und die Weise wie wir wahrnehmen relational-logisch ordnend und zugleich affektiv wertend erfolgt (vgl. hierzu vertiefend Whithehead 2000 [1927]: 101-104). Zudem besitzt gerade der Mensch das Vermögen, beide Aspekte in bildnerische Elemente umzusetzen (und wohl auch andere Medien) und über sie das von uns Verstandene festzuhalten und zu kommunizieren. Derart vermögen wir unsere Ansichten von der Welt (bewertete und damit handlungsrelevante Informationen) einerseits zu bewahren und an Andere weiterzugeben, da auch sie diese nach den gleichen Verfahren auswerten, dabei unsere Sichtweise aufnehmen und beantworten bzw. weiterverarbeiten können. So werden konkret mit Bezug auf das illustrierte Titelblatt der Jugend über die Wahl der Mittel (Linien, Flächen, Farben auf Papier), die Art und Weise ihrer Setzung 
(durchgezogen, ausgedehnt, deckend) und über die Formen, die mit ihnen beschrieben werden (Figurinen, Baum und Strahlennetz) die Weichen für eine bestimmte Bildwirkung und damit Bildaussage gestellt, deren Bedeutung wir verstehen und zu der wir eine Haltung einnehmen können. Wie bewusst wir eine Stellung zu der Bildaussage beziehen, hängt letztlich davon ab, wie in der Praxis das Verhältnis von Form und Inhalt gesetzt wurde. Auffällige Brüche, die sich in Dissonanzen bzw. Spannungen zwischen beiden äußern, reizen den Wahrnehmungsapparat. Die plötzliche Wendung der räumlichen Ordnung von der Tiefe in den Bildvordergrund lässt sich als ein solches Moment beschreiben. So eröffnen die Analyse und die Forschungen zur Eigenlogik, alle Bilder verfolgen Zwecke, die jedoch nicht immer gleich offensichtlich werden. Von den zweckfreien, schönen Künsten zu sprechen, lässt sich insofern nicht ohne Vorbehalte ausgehen.

Abschließend gilt es festzuhalten, dass mit der Untersuchung der Mechanismen (Gestaltung), Prozesse (Wahrnehmung) und Praktiken (Verfahren der Metaisierung) zur Generierung von Bedeutung durch bzw. im Medium Bild, ein Austausch von Ansichten des Produzenten und Rezipienten sichtbar wird, der als ein dialogisches Verfahren beschrieben werden kann. Im Verbund mit Texten, so die Annahme, wird dieses Moment potenziert. Deren charakteristische Eigenlogik und reflexiv verarbeitbaren Sinnpotentiale treffen auf die, über das Bild vermittelten. Es ist wohl anzunehmen, wie es sich mir aus bildwissenschaftlicher Perspektive vermittelt und von den Forschungsansätzen bestätigt wird, dass dieses Zusammentreffen und weiterführend, das wechselseitige Verstehen sich auch mit Bezug auf Texte nicht nur an den feststellbaren begrifflichen Inhalten, sondern auch auf der Ebene der Eigenlogik entzündet (vgl. hierzu auch Cassirer 1964 [1923] und Humboldt 1843 [1820]). Diese Annahme zu untermauern, ließe sich jedoch ausschließlich, wie weiterführende Forschungen zeigen müssten, in einem interdisziplinären Projekt verfolgen. Auf der Basis eines semiotischen Zugriffs sehe ich dafür vielversprechende Perspektiven. Die historische Zeitschriftenforschung, wie sie der vorliegende Band versteht, ist ein solches vielversprechendes Projekt, das von der besonderen Verbindung von Bild und Text Illustrierter Zeitschriften um 1900 motiviert, Forscherinnen und Forscher unterschiedlicher Disziplinen zusammenführte. Im Rahmen des Möglichen bzw. der eigenen bildwissenschaftlichen Forschungsperspektive galt es mit der vorgelegten Untersuchung, die spezifischen bildlichen Voraussetzungen zur Generierung von Bedeutung innerhalb des angenommenen Kommunikationsund Kulturmodells aufzuzeigen. 


\section{LITERATUR}

Bauer, Hermann (1988 [1985]): Form, Struktur, Stil: Die formanalytischen und formgeschichtlichen Methoden. In: Belting, Hans / Dilly, Heinrich / Kemp, Wolfgang / Sauerländer, Willibald / Warnke, Martin (Hg.): Kunstgeschichte. Eine Einführung. Berlin: Reimer. S. 151-168.

Boehm, Goffried (1985): Zu einer Hermeneutik des Bildes. In: Gadamer, HansGeorg / Boehm, Gottfried (Hg.): Seminar: Die Hermeneutik und die Wissenschaften (1978). Frankfurt a.M.: Suhrkamp. S. 444-47.

Boehm, Gottfried (1980): Bildsinn und Sinnesorgane. In: Neue Hefte für Philosophie 18/19 (1980): Anschauung als ästhetische Kategorie. S. 118-132.

Boehm, Gottfried (1987): Bild und Zeit. In: Pfaflik, Hannelore (Hg.): Das Phänomen Zeit in Kunst und Wissenschaft. Weinheim: VCH. S. 1-23.

Boehm, Gottfried (1992): Sehen. Hermeneutische Reflexionen. In: Figal, Günter / Rudolph, Enno (Hg.): Internationale Zeitschrift für Philosophie 1 (1992). S. 5067.

Boehm, Gottfried (2007): Unbestimmtheit. Zur Logik des Bildes. In: Ders.: Wie Bilder Sinn erzeugen. Die Macht des Zeigens. Berlin: Berlin University Press. S. 199-212.

Boehm, Gottfried (2008): Augenmaß. Zur Genese der ikonischen Evidenz. In: Boehm, Gottfried / Mersmann, Birgit / Spies, Christian (Hg.): Movens Bild. Zwischen Evidenz und Affekt. München: Fink. S. 15-38.

Böhme, Hartmut (1997): Aby M. Warburg (1866-1929). In: Michaels, Axel (Hg.): Klassiker der Religionswissenschaft. Von Friedrich Schleiermacher bis Mircea Eliade. München: Beck. S. 133-157. URL: http://www.culture.hu-berlin.de/hb/ static/archiv/volltexte/pdf/Warburg.pdf. S. 1-38. (letzter Zugriff: 29.04.2016).

Cassirer, Ernst (1964 [1923]): Philosophie der Symbolischen Formen, Bd. I. Die Sprache. Darmstadt: Wissenschaftliche Buchgesellschaft.

Cassirer, Ernst (1964 [1929]): Philosophie der Symbolischen Formen, Bd. III. Phänomenologie der Erkenntnis. Darmstadt: Wissenschaftliche Buchgesellschaft.

Cassirer, Ernst (2007 [1944]): Versuch über den Menschen, Einführung in eine Philosophie der Kultur. [Orig.: An Essay on Man] Hamburg: Meiner.

Cesare, Guiseppe di / Di Dio, Cinzia / Rochat, Magali J. / Sinigaglia, Corrado / Bruschweiler-Stern, Nadia / Stern, Daniel N. / Rizzolatti, Giacomo (2013): The neural correlates of $»$ vitality form $«$ recognition: an fMRI study. In: Social Cognitive and Affective Neuroscience (June 17, 2013). S. 1-10. URL: http://scan. oxfordjournals.org/content/early/2013/06/17/scan.nst068.full.pdf+html (letzter Zugriff: 29.04.2016).

Fiedler, Konrad (1991): Über den Ursprung der künstlerischen Tätigkeit (1887). In: Boehm, Gottfried (Hg.): Konrad Fiedler, Schriften zur Kunst, Bd. I. München: Fink. S. 112-220. 
Frank, Gustav (2008): Pictorial und Iconic Turn. Ein Bild von zwei Kontroversen. In: Mitchell, W. J. T.: Bildtheorie. Hg. und mit einem Nachwort von Gustav Frank. Frankfurt a.M.: Suhrkamp. S 445-487.

Gallese, Vittorio / Freedberg, David (2007): Motion, emotion and empathy in esthetic experience. In: TRENDS in Cognitive Sciences 11.5 (2007). S. 197-203. URL: http://www.unipr.it/arpa/mirror/pubs/pdffiles/Gallese/Freedberg-Gallese \%202007.pdf (letzter Zugriff: 29.04.2016).

Hauthal, Janine / Nadj, Julijana / Nünning, Ansgar / Peters, Henning (Hg.) (2007): Metaisierung in Literatur und anderen Medien. Theoretische Grundlagen - Historische Perspektiven - Metagattungen - Funktionen. Berlin, New York: de Gruyter.

Humboldt, Wilhelm von (1848): Über das vergleichende Sprachstudium in Beziehung auf die verschiedenen Epochen der Sprachentwicklung (1820). In: Ders.: Gesammelte Werke, Bd. 3. Berlin: Reimer. S. 241-268.

Imdahl, Max (1987): Farbe, Kunsttheoretische Reflexionen in Frankreich. München: Fink.

Kant, Immanuel (1991 [1790]): Kritik der Urteilskraft. Stuttgart: Reclam.

Kaschnitz-Weinberg, Guido von (1963 [1929]): Alois Riegl. Spätrömische Kunstindustrie. In: Sedlmayr, Hans (Hg.): Hefte des kunsthistorischen Seminars der Universität München, Bd. 4: Riegls Erbe. München: Selbstverlag. S. 25-39.

Krois, John M. (1987): Cassirer. Symbolic Forms and History. New Haven, London: Yale University.

Krois, John, M. (2011 [1999]): Cassirer's »Prototype and Model« of Symbolism. Its Sources and Significance. In: Bredekamp, Horst / Lauschke, Marion (Hg.): John M. Krois. Bildkörper und Körperschema. Schriften zur Verkörperungstheorie ikonischer Formen. Berlin: Akademie. S. 44-63.

Krois, John M. (2011 [2006]): Für Bilder braucht man keine Augen. Zur Verkörperungstheorie des Ikonischen. In: Bredekamp, Horst / Lauschke, Marion (Hg.): John M. Krois. Bildkörper und Körperschema. Schriften zur Verkörperungstheorie ikonischer Formen. Berlin: Akademie. S. 132-161.

Krois, John M. (2011 [2007]): Synesthesia and the Theory of Signs. In: Bredekamp, Horst / Lauschke, Marion (Hg.): John M. Krois. Bildkörper und Körperschema. Schriften zur Verkörperungstheorie ikonischer Formen. Berlin: Akademie. S. 162-175.

Krois, John M. (2011 [2009]): Image Science and Embodiment or: Peirce as Image Scientist. In: Bredekamp, Horst / Lauschke, Marion (Hg.): John M. Krois. Bildkörper und Körperschema. Schriften zur Verkörperungstheorie ikonischer Formen. Berlin: Akademie. S. 194-209.

Krois, John M. (2011 [2010a]): Experiencing Emotion in Depictions. Being Moved without Motion? In: Bredekamp, Horst / Lauschke, Marion (Hg.): John M. Krois. Bildkörper und Körperschema. Schriften zur Verkörperungstheorie ikonischer Formen. Berlin: Akademie. S. 232-251. 
Krois, John M. (2011 [2010b]): Tastbilder. Zur Verkörperungstheorie ikonischer Formen. In: Bredekamp, Horst / Lauschke, Marion (Hg.): John M. Krois. Bildkörper und Körperschema. Schriften zur Verkörperungstheorie ikonischer Formen. Berlin: Akademie. S. 210-231.

Krois, John M. (2011a): Bildkörper und Körperschema. In: Bredekamp, Horst / Lauschke, Marion (Hg.): John M. Krois. Bildkörper und Körperschema. Schriften zur Verkörperungstheorie ikonischer Formen. Berlin: Akademie. S. 252271.

Krois, John M. (2011b): Enactivism and Embodiment in Picture Acts. The Chirality of Images. In: Bredekamp, Horst / Lauschke, Marion (Hg.): John M. Krois. Bildkörper und Körperschema. Schriften zur Verkörperungstheorie ikonischer Formen. Berlin: Akademie. S. 272-289.

Krois, John M. (2011c): Was sind und was wollen Bilder? In: Bredekamp, Horst / Lauschke, Marion (Hg.): John M. Krois. Bildkörper und Körperschema. Schriften zur Verkörperungstheorie ikonischer Formen. Berlin: Akademie. S. 290306.

Langer, Susanne K. (1965 [1942]): Philosophie auf neuem Wege. Das Symbol im Denken, im Ritus und in der Kunst. Engl. Philosophy in a New Key. Berlin: Fischer.

Langer, Susanne K. (1967 [1953]): Feeling and Form. A Theory of Art Developed from a Philosophy in a New Key. London: Routledge \& Kegan Paul.

Langer, Susanne K. (1985 [1965]): Mind. An Essay on Human Feeling. Vol. 1. Baltimore, London: John Hopkins University.

Langer, Susanne K. (1972): Mind. An Essay on Human Feeling. Vol. 2. Baltimore, London: John Hopkins University.

Panofsky, Erwin (1984 [1932]): Zum Problem der Beschreibung und Inhaltsdeutung von Werken der bildenden Kunst. In: Kaemmerling, Ekkehard (Hg.): Bildende Kunst als Zeichensystem 1. Ikonographie und Ikonologie. Theorien Entwicklung - Probleme. Köln: Dumont. S. 185-225.

Panofsky, Erwin (1984 [1939]): Ikonographie und Ikonologie. In: Kaemmerling, Ekkehard (Hg.): Bildende Kunst als Zeichensystem 1. Ikonographie und Ikonologie. Theorien - Entwicklung - Probleme. Köln: Dumont. S. 185-225.

Peirce, Charles S. (1993 [1903]): Phänomen und Logik der Zeichen. Engl. Syllabus of Certain Topics of Logic. In: Pape, Helmut (Hg.): Charles S. Peirce, Phänomen und Logik der Zeichen. Frankfurt a.M.: Suhrkamp.

Prange, Regine (2004): Die Geburt der Kunstgeschichte. Philosophische Ästhetik und empirische Wissenschaft. Köln: Deubner.

Riegl, Alois (1901): Die spätrömische Kunstindustrie nach den Funden in Österreich-Ungarn. Heidelberg: Universitätsbibliothek. URL: http://digi.ub.uniheidelberg.de/diglit/riegl1901 (letzter Zugriff 29.04.2016).

Sauer, Martina (2009): Wahrnehmen von Sinn vor jeder sprachlichen oder gedanklichen Fassung? Frage an Ernst Cassirer. In: Kunstgeschichte. Open Peer Re- 
viewed Journal (2009). URL: http://www.kunstgeschichte-ejournal.net/63/ (letzter Zugriff: 29.04.2016).

Sauer, Martina (2011): Entwicklungspsychologie/Neurowissenschaft und Kunstgeschichte - Ein Beitrag zur Diskussion von Form als Grundlage von Wahrnehmungs- und Gestaltungsprinzipien. In: Kunstgeschichte. Open Peer Reviewed Journal: URL: http://www.kunstgeschichte-ejournal.net/134/ (letzter Zugriff: 10.11.2014).

Sauer, Martina (2012): Faszination - Schrecken. Zur Handlungsrelevanz ästhetischer Erfahrung anhand Anselm Kiefers Deutschlandbilder, Heidelberg: Universitätsbibliothek URL: http://archiv.ub.uni-heidelberg.de/artdok/1851/ (letzter Zugriff: 29.04.2016).

Sauer, Martina (2014): Cézanne, van Gogh, Monet. Genese der Abstraktion, (Diss. Basel 1989). Heidelberg: Universitätsbibliothek. URL: http://archiv.ub.uniheidelberg.de/artdok/2573/ (letzter Zugriff: 29.04.2016).

Sauer, Martina (2014): Ästhetik und Pragmatismus. Zur funktionalen Relevanz einer nicht-diskursiven Formauffassung bei Cassirer, Langer und Krois. In: IMAGE. Zeitschrift für interdisziplinäre Bildwissenschaft 20 (2014). S. 49-69. URL: http://www.gib.uni-tuebingen.de/image?function=fnArticle\&showArticle $=303$ (letzter Zugriff: 29.04.2016).

Sauer, Martina (2015): Visualität und Geschichte. Bilder als historische Akteure im Anschluss an Verkörperungstheorien. In: Grüne, Niels / Oberhauser, Claus (Hg.): Jenseits des Illustrativen. Visuelle Medien und Strategien politischer Kommunikation. Göttingen: V\&R unipress. S. 39-60.

Schade, Sigrid / Wenk, Silke (2011): Studien zur visuellen Kultur. Einführung in ein transdisziplinäres Forschungsfeld. Bielefeld: transcript.

Sedlmayr, Hans (1985): Verlust der Mitte. Die bildende Kunst des 19. und 20. Jahrhunderts als Symptom und Symbol der Zeit (1948). Frankfurt a.M., Berlin, Wien: Ullstein.

Stern, Daniel N. (1992 [1986]): Die Lebenserfahrung des Säuglings. Stuttgart, New York: Klett-Cotta.

Stern, Daniel N. (2011 [2010]): Ausdrucksformen der Vitalität. Die Erforschung dynamischen Erlebens in Psychotherapie, Entwicklungspsychologie und den Künsten. Frankfurt a.M.: Brandes \& Apsel.

Warburg, Aby M. (1992 [1923]): Schlangenritual. Ein Reisebericht. Mit einem Nachwort von Ulrich Raulff. Berlin: Wagenbach.

Werner, Heinz (1959 [1926]): Einführung in die Entwicklungspsychologie. München: Johann Ambrosius Barth.

Wiesing, Lambert (2008 [1997]): Die Sichtbarkeit des Bildes. Geschichte und Perspektiven der formalen Ästhetik. Frankfurt a.M., New York: Campus.

Wiesing, Lambert (2013): Sehen lassen. Die Praxis des Zeigens. Berlin: Suhrkamp.

Whitehead, Alfred North (2000 [1927]): Kulturelle Symbolisierung. In: Lachmann, Rolf (Hg.): Kulturelle Symbolisierung. Frankfurt a.M.: Suhrkamp. 
Wölfflin, Heinrich (1923 [1915]): Kunstgeschichtliche Grundbegriffe. Das Problem der Stilentwicklung in der neueren Kunst. München: Bruckmann.

Zimmermann, Robert (1854): Die speculative Ästhetik und die Kritik. In: Österreichische Blätter für Literatur und Kunst. Wien: Gehlen. S. 37-40. 